\title{
Ein Spiegel der Entwicklungen in der stationären Pädiatrie
}

Bernhard Frey, Vera Bernet, Vincenzo Cannizzaro

Die Autoren haben keine Interessenverbindungen, die für diesen Artikel relevant sind.

Korrespondenz:

Prof. Dr. med. Bernhard Frey Abteilung für Intensivmedizin und Neonatologie Universitäts-Kinderspital Steinwiesstrasse 75 CH-8032 Zürich Tel. 0442667359 Fax 0442667168 bernhard.frey[at]kispi.uzh.ch Felix H. Sennhauser,

Anhand der Entwicklung der Anzahl Todesfälle auf der neonatologisch-pädiatrischen Intensivstation lassen sich Rückschlüsse ziehen auf Veränderungen von Patientencharakteristika und Betreuung in der stationären Pädiatrie.

Dies soll exemplarisch für die Intensivmedizin am Universitäts-Kinderspital Zürich gezeigt werden. Es handelt sich um eine interdisziplinäre Intensivstation (IPS) mit 18 Plätzen. Das Altersspektrum reicht von der Geburt bis zum 16. Lebensjahr. Mit Ausnahme der Patienten mit Lebertransplantation bei schwerem Leberversagen werden alle Patienten betreut, insbesondere auch kardiologische/kardiochirurgische Patienten.

Die Entwicklung der MDSi-Daten (Minimal Dataset Intensivmedizin [1]) über die letzten 10 Jahre zeigt folgende Haupttrends (Abb. 1): Abnahme der absoluten Zahl an Todesfällen auf der IPS, Zunahme des Krankheits-Schweregrads respektive der erwarteten Mortalität bei Eintritt auf die IPS (PIM2, Paediatric Index of Mortality [2]), Abnahme der prozentualen (beobachteten) IPS-Mortalität.

Es gibt also klare Hinweise, dass heute weniger Kinder auf der Intensivstation sterben als früher. Dafür gibt es folgende Gründe: Einführung der Palliativmedizin, weniger schwerverletzte Kinder, weniger Neugeborene mit schweren Fehlbildungen, erfolgreiche Behandlung von schwerkranken Kindern, die früher gestorben sind. Früher wurde fast jedes Kind

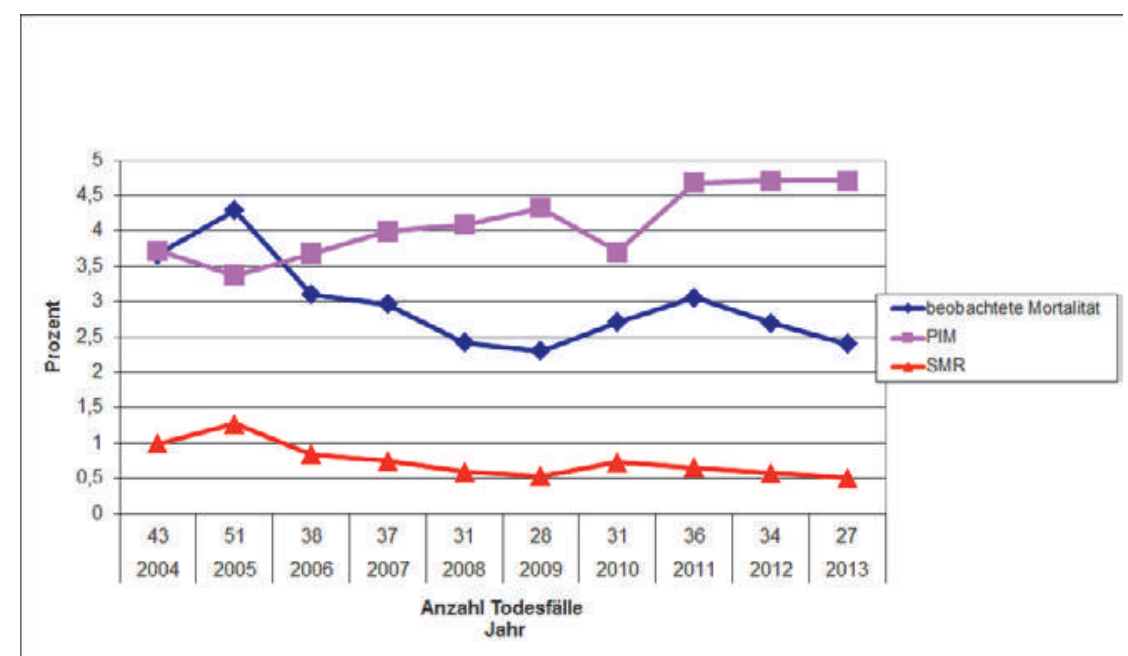

\section{Abbildung 1}

Beobachtete und «erwartete» Mortalität, Intensivstation Universitäts-Kinderspital Zürich (PIM: Paediatric Index of Mortality = «erwartete» Mortalität; SMR: Standardised Mortality Ratio = beobachtete/erwartete Mortalität): sinkende Mortalität trotz zunehmendem Schweregrad.

\section{Mortalité et soins intensifs pédiatriques}

L'analyse des données minimales pour la médecine intensive (MDSi) du service de néonatologie et des soins intensifs pédiatriques de l'Hôpital des enfants de Zurich au cours des dix dernières années montre une augmentation continue du degré de gravité des patients pris en charge et une diminution de la mortalité absolue et relative. Cette évolution reflète une concentration accrue de la pédiatrie hospitalière sur les cas aigus sévères ainsi que l'introduction de la médecine palliative et de la prise de décision éthique. En effet, une partie des enfants en phase terminale ne font aujourd'hui plus l'objet de mesures intensives lourdes et inutiles, mais sont pris en charge dans leur département usuel ou à domicile. La possibilité de pouvoir recourir à des modalités de traitement intensif hautement invasives confronte l'équipe de soins à des exigences éthiques élevées.

im terminalen Stadium auf die Intensivstation verlegt und starb dort. Die Einführung der Palliativmedizin und eines strukturierten Modells der ethischen Entscheidungsfindung führten zur positiven Entwicklung, dass ein Teil der Kinder im terminalen Stadium nicht mehr den unnötigen und belastenden intensivmedizinischen Massnahmen ausgesetzt wird, sondern auf der üblichen Bettenstation oder zu Hause gepflegt und betreut wird [3, 4]. Nach wie vor ist die Sterbebegleitung für Pflegende und Ärzte der Kinder-Intensivstation ein wichtiger Bereich, sei es bei Kindern, die akut erkrankten und primär eine umfassende lebenserhaltende Therapie erhielten oder beim begründeten Abbruch von intensivmedizinischen Therapiemassnahmen.

Am Universitäts-Kinderspital Zürich zeigte sich in den letzten Jahren ein relevanter Rückgang an schwerverletzten Kindern mit Polytrauma oder Schädel-Hirn-Trauma. Diese erfreuliche Entwicklung könnte unter anderem bedingt sein durch präventive Massnahmen wie Tempobeschränkungen im Verkehr und das Tragen von Helmen. Durch die verbesserte pränatale Diagnostik kommt es seltener vor, dass Kinder mit letalen Fehlbildungssyndromen (z. B. Trisomie 13 oder 18) bis zur Geburt ausgetragen werden. Schliesslich ist die absolute Abnahme an Todesfällen auf der Intensivstation sicher auch auf 
neue und verbesserte Behandlungsmethoden zurückzuführen. Ein Beispiel dafür ist die extrakorporelle Membranoxygenierung (ECMO). Dank dem Einsatz von ECMO konnte in den vergangenen Jahren das Leben von Kindern gerettet werden, die ohne diese Therapie gestorben wären: kardiale Patienten, Neugeborene mit persistierendem fetalen Kreislauf, Kinder mit Atemversagen bei Pneumonie oder mit Kreislaufversagen bei septischem Schock. Die Möglichkeit, auf solche hochinvasiven Therapiemodalitäten zurückgreifen zu können, stellt an die Behandlungsteams grosse ethische Anforderungen. So darf die ECMO-Therapie nur initialisiert bzw. fortgeführt werden, wenn eine gute Langzeitprognose vorliegt, z.B. erwartete Reversibilität des Grundleidens oder allenfalls Überbrückung zur möglichen Herz-Transplantation. Auf keinen Fall darf durch die ECMOTherapie das Leiden des Kindes unnötig verlängert werden. Im Zusammenhang mit den neu verfügbaren invasiven Therapieoptionen muss festgehalten werden, dass kein grundsätzlicher Anspruch auf die Durchführung von medizinisch nicht indizierten,

\section{«Ein Teil der Kinder im terminalen Stadium wird nicht mehr den unnötigen und belastenden intensivmedizinischen Massnahmen ausgesetzt.»}

weil wirkungslosen oder aussichtslosen Therapien besteht [5].

Die Abnahme der IPS-Mortalität bzw. die kontinuierliche Verbesserung des Verhältnisses zwischen beobachteter und berechneter Mortalität (Standardisierte Mortalität, SMR; Abb. 1) geht auch einher mit der Einführung diverser organisatorischer Veränderungen, z.B. standardisierte Behandlungsrichtlinien, Einführung der «In-house»-Präsenz von intensivmedizinischen Kaderärzten auch im Nachtdienst, elektronisches Verordnungssystem, klare Regelung der zielführenden Zusammenarbeit mit anderen Fachdisziplinen sowie Einführung und Etablierung einer breit abgestützten Sicherheitskultur mittels Critical Incident Monitoring und Morbidity-Mortality-Konferenzen [6, 7]. Ob es sich hier lediglich um eine Assoziation oder aber eine Kausalität handelt, ist nicht klar. Alternativ muss für die beobachtete Abnahme der Standardisierten Mortalität auch eine Veränderung in der Selektion der Patienten für den IPS-Aufenthalt in Betracht gezogen werden [8]. Kürzlich wurde darum eine Neukalibrierung des PIM publiziert (PIM3) [9].

Eine weitere Auffälligkeit ist die stete Zunahme des Krankheits-Schweregrades bei IPS-Eintritt, gemessen mit dem "Paediatric Index of Mortality» (PIM2). Dieser Score errechnet eine sogenannt «erwartete Mortalität» bei Übernahme des Patienten auf die IPS und basiert auf verschiedenen pathophysiologischen und diagnostischen Parametern. Ein wahrscheinlicher Hauptgrund für die Zunahme des Schweregrades bei IPS-Eintritt ist unseres Erachtens die zunehmende Fokussierung der stationären Pädiatrie auf hochakute Fälle, während weniger schwerkranke Kinder im ambulanten Setting (praktizierende Kinderärzte und ambulante Dienste der Kinderkliniken) frühzeitig und kompetent betreut werden. Durch Einführung von neuen Behandlungsmethoden werden auf Bettenstationen in der stationären Pädiatrie zunehmend schwerer kranke Kinder monitorisiert und betreut. Das führt dazu, dass nicht nur auf der Intensivstation, sondern auch auf den übrigen Bettenstationen der Krankheitsschweregrad der Patienten zunimmt. In dieser Situation müssen die beschränkten IPS-Plätze nach klaren Kriterien vergeben werden (künstliche Beatmung, Kreislaufunterstützung, invasives Monitoring) und es steigt somit der Bedarf für die Einrichtung von «Intermediate Care Units (IMC)» mit klaren Qualitätskriterien [10].

Die langfristige Erhebung der hier präsentierten Daten und fachübergreifende Register sind ein wichtiges exemplarisches Planungsinstrument für die zukünftige strategische und strukturelle Organisation einer situations- und bedarfsgerechten Behandlung und Betreuung von Kindern aller Altersstufen vom Neugeborenen bis zum Adoleszenten.

\section{Literatur}

1 Minimal dataset of the Swiss Society of Intensive Care Medicine. www.sgi-ssmi.ch $\rightarrow$ Qualität $\rightarrow$ MDSi.

2 Slater A, Shann F. Pearson G. PIM2: a revised version of the Paediatric Index of Mortality. Intensive Care Med. 2003;29:278-85.

3 Bergstraesser E. Pediatric palliative care - when quality of life becomes the main focus of treatment. Eur J Pediatr. 2013;172:139-50.

4 Streuli JC, Staubli G, Pfändler-Poletti M, Baumann-Hölzle R, Ersch J. Five-year experience of clinical ethics consultations in a pediatric teaching hospital. Eur J Pediatr. DOI 10.1007/s00431-013-2221-2.

5 Schweizerische Akademie der Medizinischen Wissenschaften (SAMW). Intensivmedizinische Massnahmen. Medizinisch-ethische Richtlinien und Empfehlungen. 1. Aufl. SAMW; 2013.

6 Pronovost PJ, Nolan T, Zeger S, Miller M, Rubin H. How can clinicians measure safety and quality in acute care? Lancet. 2004;363:1061-7.

7 Valentin A, Capuzzo M, Guidet B, Moreno R, Metnitz B, Bauer P, et al. Errors in administration of parenteral drugs in intensive care units: multinational prospective study. BMJ. 2009;338:b814.

8 Tibby SM, Holton F, Durward A, Murdoch IA. Is paediatric index of mortality 2 already out of date? Crit Care. 2004;8(suppl1):P329.

9 Straney L, Clements A, Parslow RC, Pearson G, Shann F, Alexander J, Slater A. Paediatric Index of Mortality 3: an updated model for predicting mortality in pediatric intensive care. Pediatr Crit Care Med. 2013;14:673-81.

10 Schweizerische Richtlinien für die Anerkennung von Intermediate-Care Units. www.sgi-ssmi.ch $\rightarrow$ Qualität $\rightarrow$ IMC. 